\title{
Smoothened-antagonists reverse homogentisic acid- induced alterations of Hedgehog signalling and primary cilium length in alkaptonuria
}

\begin{tabular}{|c|c|}
\hline Journal: & Journal of Cellular Physiology \\
\hline Manuscript ID & JCP-16-0466.R1 \\
\hline Wiley - Manuscript type: & Original Research Article \\
\hline Date Submitted by the Author: & 29-Nov-2016 \\
\hline Complete List of Authors: & $\begin{array}{l}\text { Gambassi, Silvia; Università degli Studi di Siena, Dipartimento di } \\
\text { Biotecnologie, Chimica e Farmacia } \\
\text { Geminiani, Michela; Università degli Studi di Siena, Dipartimento di } \\
\text { Biotecnologie, Chimica e Farmacia } \\
\text { Thorpe, Stephen; Queen Mary University of London, School of Engineering } \\
\text { and Materials Science } \\
\text { Bernardini, Giulia; Universita degli Studi di Siena, Dipartimento di } \\
\text { Biotecnologie, Chimica e Farmacia } \\
\text { Millucci, Lia; Universita degli Studi di Siena, Dipartimento Biotecnologie } \\
\text { Chimica e Farmacia } \\
\text { Braconi, Daniela; Universita degli Studi di Siena, Dipartimento } \\
\text { Biotecnologie, Chimica e Farmacia } \\
\text { Orlandini, Maurizio; Università degli Studi di Siena, Dipartimento di } \\
\text { Biotecnologie, Chimica e Farmacia } \\
\text { Thompson, Clare; Queen Mary University of London, School of Engineering } \\
\text { and Materials Science } \\
\text { Petricci, Elena; Universita degli Studi di Siena, Dipartimento Biotecnologie, } \\
\text { Chimica e Farmacia } \\
\text { Manetti, Fabrizio; Universita degli Studi di Siena, Dipartimento di } \\
\text { Biotecnologie, Chimica e Farmacia } \\
\text { Taddei, Maurizio; Universita degli Studi di Siena, Dipartimento } \\
\text { Biotecnologie, chimica e farmacia } \\
\text { Knight, Martin; Queen Mary, University of London, Medical Engineering } \\
\text { Division, Department of Engineering } \\
\text { Santucci, Annalisa; Università degli Studi di Siena, Dipartimento di } \\
\text { Biotecnologie, Chimica e Farmacia }\end{array}$ \\
\hline Key Words: & Ochronosis, Confocal microscopy, Acetylated a-tubulin, Chondrocytes \\
\hline
\end{tabular}


Smoothened-antagonists reverse homogentisic acid-induced alterations of Hedgehog signalling and primary cilium length in alkaptonuria

Silvia Gambassi ${ }^{1}$, Michela Geminiani ${ }^{1}$, Stephen D Thorpe $^{2}$, Giulia Bernardini ${ }^{1}$, Lia Millucci $^{1}$, Daniela Braconi ${ }^{1}$, Maurizio Orlandini ${ }^{1}$, Clare L Thompson $^{2}$, Elena Petricci ${ }^{1}$, Fabrizio Manetti $^{1}$, Maurizio Taddei ${ }^{1}$, Martin M Knight ${ }^{2}$ and Annalisa Santucci ${ }^{1 *}$

${ }^{1}$ Dipartimento di Biotecnologie, Chimica e Farmacia, Università degli Studi di Siena, Siena, Italy

${ }^{2}$ Institute of Bioengineering, School of Engineering and Materials Science, Queen Mary University of London, Mile End Rd, London, E1 4NS, UK

* Corresponding author: Prof. Annalisa Santucci

Università degli Studi di Siena

Dipartimento di Biotecnologie, Chimica e Farmacia

Via Aldo Moro 2, 53100 Siena, Italy

E-mail: annalisa.santucci@unisi.it

tel: $+390577-234958$

fax: $+390577-234954$

Running head: Hedgehog signalling pathway modulation in alkaptonuria

Keywords:

- Ochronosis

- Confocal microscopy

- Acetylated $\alpha$-tubulin

- Chondrocytes

Total number of text figures: 5

Total number of text tables: 1

Contract Grant sponsor: Fondazione Telethon grant GGP10058 


\section{Abstract}

Alkaptonuria (AKU) is an ultra-rare genetic disease, in which the accumulation of a toxic metabolite, homogentisic acid (HGA) leads to the systemic development of ochronotic aggregates. These aggregates cause severe complications mainly at the level of joints with extensive degradation of the articular cartilage. Primary cilia have been demonstrated to play an essential role in development and the maintenance of articular cartilage homeostasis, through their involvement in mechanosignalling and Hedgehog signalling pathways. Hedgehog signalling has been demonstrated to be activated in osteoarthritis (OA) and to drive cartilage degeneration in vivo. The numerous similarities between $\mathrm{OA}$ and AKU suggest that primary cilia hedgehog signalling may also be altered in AKU.

Thus, we characterised an AKU cellular model in which healthy chondrocytes were treated with HGA $(66 \mu \mathrm{M})$ to replicate AKU cartilage pathology. We investigated the degree of activation of the hedgehog signalling pathway and how treatment with inhibitors of the receptor Smoothened (Smo) influenced hedgehog activation and primary cilia structure. The results obtained in this work provide a further step in the comprehension of the pathophysiological features of AKU, suggesting a potential therapeutic approach to modulate AKU cartilage degradation processes through manipulation of the hedgehog pathway. 


\section{Introduction}

Alkaptonuria (AKU) is an ultra-rare genetic metabolic disease (1:250.000 - 1.000.000), in which mutations of the enzyme homogentisate 1,2 dioxygenase (HGD) prevent it from metabolizing homogentisic acid (HGA) in the catabolic pathway of tyrosine and phenylalanine (Braconi et al., 2015). Accumulation of this toxic compound and its derivatives results in urine darkening and in the formation of pigmented protein aggregates causing ochronosis within the whole organism. For this reason, AKU is considered a multi-systemic disease, although the most common body districts severely affected by HGA accumulation are joints, as AKU patients evidence serious movement impairments resulting in an ochronotic arthropathy. There are numerous findings indicating that, among the processes that lead to cartilage degradation in AKU, important roles are played by oxidative stress (Braconi et al., 2011; Braconi et al., 2010; Braconi et al., 2015), inflammation (Millucci et al., 2014c), and accumulation of serum amyloid A (SAA), describing AKU as a secondary amyloidosis (Millucci et al., 2015a; Millucci et al., 2014a; Millucci et al., 2014b). More recently, the structural analysis of AKU chondrocyte cytoskeleton revealed that the organization of the three main cytoskeletal proteins (tubulin, actin and vimentin) is altered in AKU cells (Geminiani et al., 2016) and that chondrocytes cultured in HGA-added medium started developing the same alterations observed in AKU chondrocytes. Alterations in cytoskeletal tension have previously been shown to regulate the structure and function of primary cilia (McMurray et al., 2013; Pitaval et al., 2010). Primary cilia are signalling organelles involved in numerous signalling pathways (for review see (Berbari et al., 2009). In articular cartilage primary cilia mediate mechanosignalling (Wann et al., 2012), inflammatory signalling (Wann et al., 2014; Wann and Knight, 2012; Wann et al., 2013) and Hedgehog (Hh) signalling (Thompson et al., 2014; Thompson et al., 2016). All have been linked to osteoarthritis, however this study concentrates on whether Hh signalling is disrupted in a cellular model of AKU. Briefly, hedgehog signalling is activated by the binding of one of the three Hh ligands (Sonic Hedgehog, Shh; Indian Hedgehog, Ihh; Desert Hedgehog, Dhh) to the receptor located on the cellular membrane, Patched 1 (Patch-1). This enables the trafficking and localization on the primary cilium of a seven-transmembrane-span receptor-like protein, Smoothened (Smo) (Ingham and McMahon, 2001). This then leads to the activation of the transcriptional activators, Gli-1 and Gli-2, and suppression of the transcriptional repressor, Gli-3. Studies from Knight's group have shown that Hh signalling is modulated by changes in primary cilia length induced by mechanical loading (Thompson et al., 2014) or lithium chloride (Thompson et al., 2016).

A relationship between the activation of the Hh pathway and osteoarthritis (OA) has previously been described in vivo (Akhtar et al., 2015; Lin et al., 2009; Wei et al., 2012) although other in vitro 
studies show that Hh signalling alone does not directly activate cartilage degradation (Thompson et al., 2015). Hh signalling is also implicated in the regulation of chondrocytes growth and differentiation (St-Jacques et al., 1999). The numerous similarities between AKU and OA pathogenesis prompted us to investigate the effects of Hh signalling pathway modulation in AKU. In the present study we therefore investigated the potential to regulate hedgehog signalling in healthy and HGA-treated human chondrocytes. For these studies we used four Smo-antagonists, namely cyclopamine, vismodegib and two other recently described antagonists, MRT89 and MRT61 (Hoch et al., 2015; Solinas et al., 2012). We measured the response in terms of the expression of the constitutive activator of Hh signalling, Gli-1 and the length of the primary cilia. The aim of this study was therefore to examine primary cilia length in articular chondrocytes isolated from AKU patients and to set-up an AKU cell model in order to analyse the activation of the Hh signalling pathway and its inhibition by means of specific antagonists of the receptor Smo. 


\section{Materials and methods}

\section{Anti-Smo compounds}

The Smo agonist SAG was purchased from Cayman Chemical (Michigan, USA), vismodegib from Santa Cruz Biotechnology (Dallas, Texas, USA) and cyclopamine, MRT89 and MRT61 were synthesized in our laboratories as previously reported by us (Hoch et al., 2015; Manetti et al., 2010; Roudaut et al., 2011; Solinas et al., 2012). These compounds were selected from among a small library of available compounds, on the basis of previous experiments in which their inhibition activity against Smo was determined (data not shown). All the testing compounds are listed in Table 1SM.

\section{Chondrocyte isolation, culture and treatment}

Primary human articular chondrocytes were isolated from articular cartilage samples by means of enzymatic extraction, as previously described (Braconi et al., 2012). All the samples were obtained from consenting AKU patients or patients not affected from rheumatic pathologies following traumatic accident surgeries. The experiments performed in this study were conducted according to the principles of the Declaration of Helsinki $\left(64^{\text {th }}, 2013\right)$ and received approval from the Local Ethics Committee.

Primary human articular chondrocytes were cultured in high glucose Dulbecco's modified Eagle Medium (DMEM), 10\% fetal bovine serum (FBS) and 1\% Penicillin/Streptomycin (P/S) at $37{ }^{\circ} \mathrm{C}$, $5 \% \mathrm{CO}_{2}$. To reproduce in vitro the phenotypic and structural features of AKU chondrocytes, healthy chondrocytes were treated for two weeks with $66 \mu \mathrm{M}$ HGA (Sigma Aldrich, St Louis, MO). Upon reaching confluence, cells were serum-starved $(0.5 \% \mathrm{FBS})$ for $24 \mathrm{~h}$ before treatments with SAG and/or Smo antagonists cyclopamine, vismodegib, MRT89 and MRT61.

For immunofluorescence analysis, chondrocytes were seeded onto coverslips in 24 well plates and, after serum starvation, were treated with Smo antagonists (1, 10 and $100 \mathrm{nM})$ for $24 \mathrm{~h}$. For Western blot analysis, cells were incubated with Smo antagonists $(1,10,100 \mathrm{nM})$ in addition to $100 \mathrm{nM}$ $\mathrm{SAG}$, in order to ensure Hh signalling pathway activation.

\section{Immunofluorescence and confocal microscopy}

For immunofluorescence analysis of primary cilia, confluent chondrocytes were fixed with 4\% PFA for $10 \mathrm{~min}$, permeabilized with $0.5 \%$ Triton-X-100 and incubated for $45 \mathrm{~min}$ in the blocking solution (5\% goat serum). For detection of primary cilia, mouse anti-acetylated tubulin, clone 611B-1 (1:1000, Sigma Aldrich) was used, by incubating coverslips at $4{ }^{\circ} \mathrm{C}$ overnight. After repeated 
washing in $0.1 \%$ bovine serum albumin (BSA)/phosphate buffered saline (PBS), cells were incubated with an anti-mouse, Alexa Fluor-conjugated secondary antibody (1:1000, Life Technologies, California, USA) for $1 \mathrm{~h}$ at room temperature (rt). DAPI (4',6-diamidino-2phenylindole) staining was used for nuclei detection (1 $\mu \mathrm{g} / \mathrm{mL}$, Sigma Aldrich). Following washings in $0.1 \%$ BSA/PBS, coverslips were mounted with Fluoromount G (Southern Biotech, Alabama, USA).

Imaging and quantification of cilia length was performed on a Leica TCS SP5 AOBS confocal microscope with a x63/1.4-NA lens. Sequential z-stacked sections were imaged through the entirety of the cellular profile using Z-step size of $0.5 \mu \mathrm{m}, 2 \mathrm{x}$ or $4 \mathrm{x}$ zoom and an image format of $1024 \mathrm{x}$ 1024 pixels. Z stacks were reconstructed and 2D maximum intensity projections (MIPs) were created in order to measure cilia length by means of the software Image J. Approximately 100 cilia were measured over five representative fields of view for each experimental condition.

\section{Viability assay}

To assess whether treatment with anti-Smo compounds affected cells viability, MTT [3-(4,5dimethylthiazol-2-yl)-2,5-diphenyltetrazolium bromide] assay was performed. 80\% confluent cells were incubated for $3 \mathrm{~h}$ with $1 \mathrm{mg} / \mathrm{mL}$ MTT (Sigma Aldrich). Resulting formazan crystals were dissolved with DMSO and samples were read spectrophotometrically at $550 \mathrm{~nm}$.

\section{Western blot analysis}

Protein lysates were obtained firstly by lysing cells in RIPA buffer (1\% Triton-X100, $100 \mathrm{mM}$ $\mathrm{NaCl}, 0.1 \%$ SDS, $50 \mathrm{mM}$ Tris $\mathrm{pH}$ 7.2), containing $\mathrm{Na}_{3} \mathrm{VO}_{4}$ and a protease inhibitor cocktail (Sigma Aldrich), and then by sonication. Protein extracts were quantified by BCA protein assay according to the manufacturer's instructions (Thermo Fisher Scientific, Massachusetts, USA). Proteins from healthy and HGA-treated chondrocytes were separated by SDS-PAGE, loading $40 \mu \mathrm{g}$ of total protein lysate on $8 \%$ acrylamide gel. Proteins were electro-transferred onto nitrocellulose membrane ( $0.45 \mathrm{~mm}$ pore size; Whatman, Maidstone, UK), blocked for $1 \mathrm{~h}$ at $\mathrm{rt}$ in $5 \% \mathrm{BSA}$ in $0.05 \%$ Tween-20 phosphate buffered saline (PBS-T) and then incubated with anti-Gli-1 primary antibody (1:200, Santa Cruz Biotechnology) at $4{ }^{\circ} \mathrm{C}$ overnight. Membranes were washed in PBS-T and then incubated with appropriate HRP-conjugated secondary antibody (1:5000, GE Healthcare, Little Chalfont, UK) for $1 \mathrm{~h}$ at rt. For GAPDH (glyceraldehyde-3-phosphate dehydrogenase) detection, membranes were incubated with anti-GAPDH antibody (1:50000, Abcam, Cambdridge, $\mathrm{UK}$ ) for $3 \mathrm{~h}$ at $\mathrm{rt}$. After three washings in PBS-T, membranes were visualized using Luminata Crescendo (Millipore, Italy) and images acquired using ImageQuant LAS4000 (GE Healthcare). 
Analysis of band intensities was performed by ImageQuant TM TL analysis software (GE Healthcare).

\section{Statistical analysis}

All statistical analyses were conducted using Graph Pad Prism 6.01. Data were analysed by oneway or two-way ANOVA with post hoc Tukeys's multiple comparisons. Data is presented as mean \pm S.E.M. resulting from three independent experiments and in all cases statistically significant differences in relation to the untreated control were indicated at $\mathrm{p}<0.05\left(^{*}\right), \mathrm{p}<0.01(* *), \mathrm{p}<$ $0.001(* * *)$ and $\mathrm{p}<0.0001(* * * *)$. 


\section{Results}

\section{HGA-induced pigmentation validated the AKU cell model}

In order to reproduce in vitro the phenotypic characteristics of AKU chondrocytes, healthy chondrocytes were maintained in culture for 14 days in a medium containing $66 \mu \mathrm{M}$ HGA. Phase contrast images evidenced that healthy chondrocytes appeared transparent (Figure 1A), while AKU chondrocytes exhibited a black pigmentation, mainly localized around the nuclei (Figure 1B). The same features were observed in cells cultured in presence of HGA (Figure 1C), with a lower pigment deposition prevalence in respect to AKU cells.

\section{AKU and HGA-treated chondrocytes exhibited shorter primary cilia and altered Hh signalling}

Primary cilia were visualised by immunofluorescence in healthy, AKU and HGA-treated chondrocytes by labelling with anti-acetylated $\alpha$-tubulin antibodies (Figure 2A). In healthy human chondrocytes the cilia length was $3.6 \pm 1.7 \mu \mathrm{m}$ (mean $\pm \mathrm{SEM}$ ). Cilia length was reduced in AKU $(2.5 \pm 1 \mu \mathrm{m})$ and HGA-treated chondrocytes $(2.7 \pm 1 \mu \mathrm{m})$ and the differences were statistically significant (Figure 2B). These studies further support the suitability of HGA treatment as a model of AKU.

Western blot analysis of Gli-1 expression in the three cell groups (Figure 2C) demonstrated that $\mathrm{Hh}$ signalling was altered in AKU and HGA-treated cells. In particular, Gli-1 protein was expressed at higher levels in the disease condition (AKU and $+\mathrm{HGA}$ ), in respect to healthy chondrocytes.

\section{Anti-Smo treatment did not affect cell viability}

Cell viability after a $24 \mathrm{~h}$ treatment with Smo antagonists was assessed by means of MTT assay. DMSO was used as control substance, since it was the dissolving agent for all the test compounds. Absorbance values at $550 \mathrm{~nm}$ were registered and results were normalised to the untreated value and given as a percentage. HGA or DMSO treatment had no statistically significant effect on cell viability compared to untreated controls (Figure 3). The assay allowed evaluation of the cytotoxicity of the antagonists of Smo in presence of HGA. Cell viability was not significantly altered after treatment with any of the four testing Smo antagonists at concentrations of 1-100 nM.

\section{Primary cilia elongated following anti-Smo treatments}

Immunofluorescence assay was performed on primary human chondrocytes cultured onto coverslips and treated for $24 \mathrm{~h}$ with anti-Smo compounds at three different concentrations $(1,10$ 
and $100 \mathrm{nM}$ ), in the presence of $66 \mu \mathrm{M}$ HGA. Confocal imaging and cilia length measurement revealed that after treatment with Smo antagonists primary cilia lengths assumed values comparable to that of normal cells in a concentration-dependent manner (Figure 4). Table 1 shows the mean \pm S.E.M cilia length and the percentage difference calculated in respect to HGA-treated cells in the absence of any Smo antagonist. The greatest cilia lengths values were obtained after treatment with either cyclopamine or vismodegib at $100 \mathrm{nM}$. In the presence of HGA, treatment with these Smo antagonists, even at lower concentration of 1 and $10 \mathrm{nM}$, was sufficient to restore the primary cilia to lengths seen in normal cells such that there were no statistically significant differences. Chondrocytes treated with MRT89 and MRT61 also showed primary cilia close to lengths values calculated in control cells, at $100 \mathrm{nM}$. In particular, MRT89 appeared to be more effective than MRT61 in restoring cilia length.

\section{Anti-Smo treatment reduced Hedgehog signalling activation}

The expression of Gli-1 protein was evaluated in order to quantify the activation of the $\mathrm{Hh}$ signalling pathway in our treated and untreated cell groups. In all cases, the pathway was stimulated by the agonist SAG $(100 \mathrm{nM})$ to amplify the level of expression of the protein. By means of western blot analysis, Gli-1 expression was higher in HGA-treated chondrocytes compared to healthy cells and decreased following a $24 \mathrm{~h}$ treatment with anti-Smo compounds (Figure 5A). Band quantification confirmed that the differences were statistically significant in a concentrationdependent manner and that in all cases it was possible to restore the expression of Gli-1 to the normal levels (Figure 5B). In particular, cyclopamine, vismodegib and the analogue MRT61 were also found to be effective at the lowest concentrations (1 and $10 \mathrm{nM})$. 


\section{Discussion}

AKU replicates aspects of joint pathology seen in more common rheumatic diseases, such as OA and rheumatoid arthritis (Tinti et al., 2010a). Degeneration of articular cartilage, loss of joint function, synovial inflammation, sub-chondral bone thickening, osteophyte formation, degeneration of ligaments in the knee and menisci, as well as chondroptosis, a peculiar type of cell death observed in cartilage, are features shared between OA and AKU (Millucci et al., 2015b).

Although AKU is a well-described condition from a clinical point of view, the molecular bases of the disease are still quite obscure. In particular, the difficulty of collecting ochronotic samples, which require very invasive techniques, together with the fact that ochronosis often causes severe damage to tissues, make the collection of suitable samples from biopsies more problematic. Consequently, the difficulty to obtain human samples for experimental studies has led to the necessity to design alternative methods to study AKU pathophysiology. Numerous findings evidence that the toxic metabolite HGA is the main responsible of the physio-pathological features of AKU (Geminiani et al., 2016; Millucci et al., 2015b; Tinti et al., 2010b), since it accumulates systematically generating ochronotic aggregates together with amyloid deposits (Millucci et al., 2014c). In this work, we demonstrated that, by adding a $66 \mu \mathrm{M}$ solution of HGA to the culture medium of primary isolated human chondrocytes, a black pigmentation was visible within cells by phase contrast imaging, entirely reproducing the phenotype of alkaptonuric chondrocytes. A further confirmation that HGA is responsible for the pathologic phenotype of AKU chondrocytes derived from the immunofluorescent assay of primary cilia in the three cellular groups analysed. Acetylated $\alpha$-tubulin staining revealed that primary cilia were shorter in AKU and HGA-treated chondrocytes in agreement with recent findings on cytoskeleton alterations in AKU. We have recently demonstrated that the cytoskeleton markers $\beta$-tubulin, actin and vimentin were severely altered in AKU chondrocytes and that HGA treatment reproduced the same ochronotic phenotype in healthy cells (Geminiani et al., 2016). These findings have prompted further investigation into AKU associated changes in the cytoskeletal structure known as the primary cilium and the functional implications. Numerous experimental results demonstrated that this organelle is fundamental for several cellular processes (Berbari et al., 2009; Ruhlen and Marberry, 2014) and that in cartilage it has a fundamental role in mechanosignalling (Wann et al., 2012), inflammatory signalling (Wann et al., 2014; Wann and Knight, 2012; Wann et al., 2013) and hedgehog signalling (Haycraft and Serra, 2008; Thompson et al., 2014; Thompson et al., 2016).

Hedgehog signalling has an important role in skeleton development (Yang et al., 2015) and acts to regulate cell differentiation, proliferation and survival in many developmental processes (Day and 
Yang, 2008). Previous studies on osteoarthritic cartilage showed that Hh signalling is activated in OA and promotes cartilage degeneration (Lin et al., 2009; Tchetina et al., 2005). In this study, we have investigated the activation of the $\mathrm{Hh}$ pathway, by focusing on the expression of the transcriptional activator of the Hh signal (Gli-1). We have demonstrated that, by stimulating the pathway in healthy and HGA-treated chondrocytes with the agonist SAG, introduced a greater induction of Gli-1 expression in the presence of HGA. To explain these results, we can hypothesize that the activity of the agonist SAG synergizes with HGA to activate Smo, although this mechanism has not been clarified yet.

Several recent studies have highlighted the potential efficacy of hedgehog antagonists in the prevention of cartilage degradation in OA and RA (Lin et al., 2009; Ruiz-Heiland et al., 2010; Zhou et al., 2014). On the basis of this previous evidence, antagonists of Smo have been tested in this work to evaluate their potential inhibitory effect of Hh pathway. In addition to the natural teratogenic compound cyclopamine, another inhibitor of Smo signalling has been used, vismodegib, which is the first antagonist of Hedgehog pathway approved by FDA for treating BBC (Basal Cell Carcinoma) (Amakye et al., 2013). Moreover, two new Smo inhibitors, MRT89 and MRT61, recently developed in our laboratories, have been tested for the same experiments. We have demonstrated that in primary human chondrocytes, the reduction in cilia length caused by treatment with HGA was restored to that in healthy cells by Smo antagonists. Cilia elongation induced by Smo antagonists has not previously been reported and the mechanism for this is unknown. Smo antagonists also reduced Gli-1 expression in a concentration-dependent manner.

The possibility to antagonize Hedgehog signalling in AKU is an important discovery, which suggest employing this pathway as a potential therapeutic target for reducing cartilage degradation processes that characterize the disease. AKU is a genetic disease, caused by mutations of the enzyme responsible for the metabolism of HGA and in most cases, the symptoms appear in adulthood, therefore targeting a cellular pathway fundamental for development processes would be useful at the later stages of the disease as a symptomatic approach to prevent further cartilage degradation caused by HGA.

\section{Conflict of interest}

None to declare

\section{Acknowledgments}

This work was supported by Telethon Italy grant GGP10058. The authors thank the Associazione Italiana Malati di Alcaptonuria (AimAKU) (grant ORPHA263402). We also thank Prof. P. Mariani and Dr. D. Gambera for providing cartilage samples. 


\section{References}

Akhtar N, Makki MS, Haqqi TM. 2015. MicroRNA-602 and microRNA-608 regulate sonic hedgehog expression via target sites in the coding region in human chondrocytes. Arthritis \& rheumatology (Hoboken, NJ 67(2):423-434.

Amakye D, Jagani Z, Dorsch M. 2013. Unraveling the therapeutic potential of the Hedgehog pathway in cancer. Nature medicine 19(11):1410-1422.

Berbari NF, O'Connor AK, Haycraft CJ, Yoder BK. 2009. The primary cilium as a complex signaling center. Curr Biol 19(13):R526-535.

Braconi D, Bernardini G, Bianchini C, Laschi M, Millucci L, Amato L, Tinti L, Serchi T, Chellini F, Spreafico A, Santucci A. 2012. Biochemical and proteomic characterization of alkaptonuric chondrocytes. Journal of cellular physiology 227(9):3333-3343.

Braconi D, Bianchini C, Bernardini G, Laschi M, Millucci L, Spreafico A, Santucci A. 2011. Redox-proteomics of the effects of homogentisic acid in an in vitro human serum model of alkaptonuric ochronosis. Journal of inherited metabolic disease 34(6):1163-1176.

Braconi D, Laschi M, Taylor AM, Bernardini G, Spreafico A, Tinti L, Gallagher JA, Santucci A. 2010. Proteomic and redox-proteomic evaluation of homogentisic acid and ascorbic acid effects on human articular chondrocytes. Journal of cellular biochemistry 111(4):922-932.

Braconi D, Millucci L, Bernardini G, Santucci A. 2015. Oxidative stress and mechanisms of ochronosis in alkaptonuria. Free radical biology \& medicine 88(Pt A):70-80.

Day TF, Yang Y. 2008. Wnt and hedgehog signaling pathways in bone development. The Journal of bone and joint surgery 90 Suppl 1:19-24.

Geminiani M, Gambassi S, Millucci L, Lupetti P, Collodel G, Mazzi L, Frediani B, Braconi D, Mazzocchi B, Laschi M, Bernardini G, Santucci A. 2016. Cytoskeleton Aberrations in Alkaptonuric Chondrocytes. Journal of cellular physiology.

Haycraft CJ, Serra R. 2008. Cilia involvement in patterning and maintenance of the skeleton. Current topics in developmental biology 85:303-332.

Hoch L, Faure H, Roudaut H, Schoenfelder A, Mann A, Girard N, Bihannic L, Ayrault O, Petricci E, Taddei M, Rognan D, Ruat M. 2015. MRT-92 inhibits Hedgehog signaling by blocking overlapping binding sites in the transmembrane domain of the Smoothened receptor. Faseb J 29(5):1817-1829.

Ingham PW, McMahon AP. 2001. Hedgehog signaling in animal development: paradigms and principles. Genes \& development 15(23):3059-3087.

Lin AC, Seeto BL, Bartoszko JM, Khoury MA, Whetstone H, Ho L, Hsu C, Ali SA, Alman BA. 2009. Modulating hedgehog signaling can attenuate the severity of osteoarthritis. Nature medicine 15(12):1421-1425.

Manetti F, Faure H, Roudaut H, Gorojankina T, Traiffort E, Schoenfelder A, Mann A, Solinas A, Taddei M, Ruat M. 2010. Virtual screening-based discovery and mechanistic characterization of the acylthiourea MRT-10 family as smoothened antagonists. Molecular pharmacology 78(4):658-665.

McMurray RJ, Wann AK, Thompson CL, Connelly JT, Knight MM. 2013. Surface topography regulates wnt signaling through control of primary cilia structure in mesenchymal stem cells. Scientific reports 3:3545.

Millucci L, Braconi D, Bernardini G, Lupetti P, Rovensky J, Ranganath L, Santucci A. 2015a. Amyloidosis in alkaptonuria. Journal of inherited metabolic disease 38(5):797-805.

Millucci L, Ghezzi L, Bernardini G, Braconi D, Lupetti P, Perfetto F, Orlandini M, Santucci A. 2014a. Diagnosis of secondary amyloidosis in alkaptonuria. Diagnostic pathology 9:185.

Millucci L, Ghezzi L, Braconi D, Laschi M, Geminiani M, Amato L, Orlandini M, Benvenuti C, Bernardini G, Santucci A. 2014b. Secondary amyloidosis in an alkaptonuric aortic valve. International journal of cardiology 172(1):e121-123. 
Millucci L, Ghezzi L, Paccagnini E, Giorgetti G, Viti C, Braconi D, Laschi M, Geminiani M, Soldani P, Lupetti P, Orlandini M, Benvenuti C, Perfetto F, Spreafico A, Bernardini G, Santucci A. 2014c. Amyloidosis, inflammation, and oxidative stress in the heart of an alkaptonuric patient. Mediators of inflammation 2014:258471.

Millucci L, Giorgetti G, Viti C, Ghezzi L, Gambassi S, Braconi D, Marzocchi B, Paffetti A, Lupetti P, Bernardini G, Orlandini M, Santucci A. 2015b. Chondroptosis in alkaptonuric cartilage. Journal of cellular physiology 230(5):1148-1157.

Pitaval A, Tseng Q, Bornens M, Thery M. 2010. Cell shape and contractility regulate ciliogenesis in cell cycle-arrested cells. The Journal of cell biology 191(2):303-312.

Roudaut H, Traiffort E, Gorojankina T, Vincent L, Faure H, Schoenfelder A, Mann A, Manetti F, Solinas A, Taddei M, Ruat M. 2011. Identification and mechanism of action of the acylguanidine MRT-83, a novel potent Smoothened antagonist. Molecular pharmacology 79(3):453-460.

Ruhlen R, Marberry K. 2014. The chondrocyte primary cilium. Osteoarthritis and cartilage / OARS, Osteoarthritis Research Society 22(8):1071-1076.

Ruiz-Heiland G, Horn A, Zerr P, Hofstetter W, Baum W, Stock M, Distler JH, Nimmerjahn F, Schett G, Zwerina J. 2010. Blockade of the hedgehog pathway inhibits osteophyte formation in arthritis. Annals of the rheumatic diseases 71(3):400-407.

Solinas A, Faure H, Roudaut H, Traiffort E, Schoenfelder A, Mann A, Manetti F, Taddei M, Ruat M. 2012. Acylthiourea, acylurea, and acylguanidine derivatives with potent hedgehog inhibiting activity. Journal of medicinal chemistry 55(4):1559-1571.

St-Jacques B, Hammerschmidt M, McMahon AP. 1999. Indian hedgehog signaling regulates proliferation and differentiation of chondrocytes and is essential for bone formation. Genes \& development 13(16):2072-2086.

Tchetina EV, Squires G, Poole AR. 2005. Increased type II collagen degradation and very early focal cartilage degeneration is associated with upregulation of chondrocyte differentiation related genes in early human articular cartilage lesions. The Journal of rheumatology 32(5):876-886.

Thompson CL, Chapple JP, Knight MM. 2014. Primary cilia disassembly down-regulates mechanosensitive hedgehog signalling: a feedback mechanism controlling ADAMTS-5 expression in chondrocytes. Osteoarthritis and cartilage / OARS, Osteoarthritis Research Society 22(3):490-498.

Thompson CL, Patel R, Kelly TA, Wann AK, Hung CT, Chapple JP, Knight MM. 2015. Hedgehog signalling does not stimulate cartilage catabolism and is inhibited by Interleukin-1beta. Arthritis research \& therapy 17:373.

Thompson CL, Wiles A, Poole CA, Knight MM. 2016. Lithium chloride modulates chondrocyte primary cilia and inhibits Hedgehog signaling. Faseb J 30(2):716-726.

Tinti L, Spreafico A, Braconi D, Millucci L, Bernardini G, Chellini F, Cavallo G, Selvi E, Galeazzi M, Marcolongo R, Gallagher JA, Santucci A. 2010a. Evaluation of antioxidant drugs for the treatment of ochronotic alkaptonuria in an in vitro human cell model. Journal of cellular physiology 225(1):84-91.

Tinti L, Taylor AM, Santucci A, Wlodarski B, Wilson PJ, Jarvis JC, Fraser WD, Davidson JS, Ranganath LR, Gallagher JA. 2010b. Development of an in vitro model to investigate joint ochronosis in alkaptonuria. Rheumatology (Oxford, England) 50(2):271-277.

Wann AK, Chapple JP, Knight MM. 2014. The primary cilium influences interleukin-1beta-induced NFkappaB signalling by regulating IKK activity. Cellular signalling 26(8):1735-1742.

Wann AK, Knight MM. 2012. Primary cilia elongation in response to interleukin-1 mediates the inflammatory response. Cell Mol Life Sci 69(17):2967-2977.

Wann AK, Thompson CL, Chapple JP, Knight MM. 2013. Interleukin-1beta sequesters hypoxia inducible factor 2alpha to the primary cilium. Cilia 2(1):17. 
Wann AK, Zuo N, Haycraft CJ, Jensen CG, Poole CA, McGlashan SR, Knight MM. 2012. Primary cilia mediate mechanotransduction through control of ATP-induced $\mathrm{Ca} 2+$ signaling in compressed chondrocytes. Faseb J 26(4):1663-1671.

Wei F, Zhou J, Wei X, Zhang J, Fleming BC, Terek R, Pei M, Chen Q, Liu T, Wei L. 2012. Activation of Indian hedgehog promotes chondrocyte hypertrophy and upregulation of MMP-13 in human osteoarthritic cartilage. Osteoarthritis and cartilage / OARS, Osteoarthritis Research Society 20(7):755-763.

Yang J, Andre P, Ye L, Yang YZ. 2015. The Hedgehog signalling pathway in bone formation. International journal of oral science 7(2):73-79.

Zhou J, Chen Q, Lanske B, Fleming BC, Terek R, Wei X, Zhang G, Wang S, Li K, Wei L. 2014. Disrupting the Indian hedgehog signaling pathway in vivo attenuates surgically induced osteoarthritis progression in Col2a1-CreERT2; Ihhfl/fl mice. Arthritis research \& therapy 16(1):R11. 


\section{Figure legends}

Figure 1. HGA-induced pigmentation by phase contrast imaging. (A) Primary human healthy chondrocytes observed at the optic microscope resulted transparent; (B) AKU chondrocytes presented pigment deposition and, similarly, healthy chondrocytes cultured in presence of HGA at $66 \mu \mathrm{M}$ showed the same phenotypic features (C). Coloured boxes represent pigmented cells, enlarged in order to show pigment deposition, mainly around nuclei. Objective magnification: 10x.

Figure 2. Primary cilia and Hh signalling characterization in primary human chondrocytes. (A) (B) Confocal imaging and statistical analysis of cilia lengths after immunostaining with anti acetylated $\alpha$-tubulin antibody. Scale bar: $20 \mu \mathrm{m}$. White boxes indicate representative cells, scale bar: $10 \mu \mathrm{m}$. Statistical analysis were performed by 2-way ANOVA with Tukey's multiple comparisons tests. (C) Western blot analysis of Gli-1 expression in response to the agonist SAG (100 nM). Statistics were performed by one-way ANOVA test. In all cases, statistically significant differences relative to untreated control were indicated.

Figure 3. MTT assay. Cell viability, expressed in percentage relative to control, was not affected neither by treatment with HGA at $66 \mu \mathrm{M}$, nor by treatment with cyclopamine, vismodegib, MRT89 and MRT61 at $1 \mathrm{nM}, 10 \mathrm{nM}$ and $100 \mathrm{nM}$.

Figure 4. Confocal imaging of primary cilia of chondrocytes treated with Smo antagonists and cilia length measurements. Images obtained at the confocal microscope show that HGA-treated chondrocytes have shorter cilia. However cilia length was restored to that seen in healthy cells by treatment with cyclopamine, vismodegib, MRT89 and MRT61 at 1, 10 and $100 \mathrm{nM}$. Data statistically analysed by 2-way ANOVA with Tukey's multiple comparisons tests (p-values calculated in comparison with CTR or +HGA samples).

Figure 5. Gli-1 expression analysis after treatment with Smo antagonists. (A) Immuno-reactive bands resulting from western blot analysis. (B) Quantification of Gli-1 expression in protein lysates obtained from primary human chondrocytes cultures in presence of HGA + SAG $(100 \mathrm{nM})+$ Smo antagonist ( $1 \mathrm{nM}, 10 \mathrm{nM}, 100 \mathrm{nM})$. Protein expression significantly decreased in a concentrationdependent manner. One-way ANOVA statistics (p-values calculated in comparison with CTR or +HGA samples). 
A

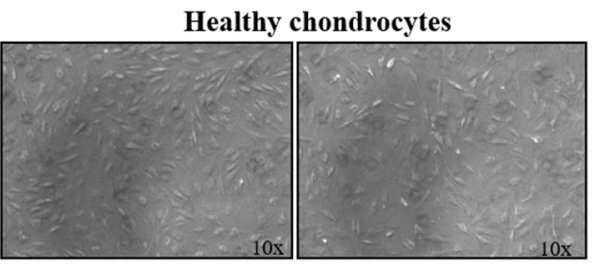

Zoomed

B AKU chondrocytes
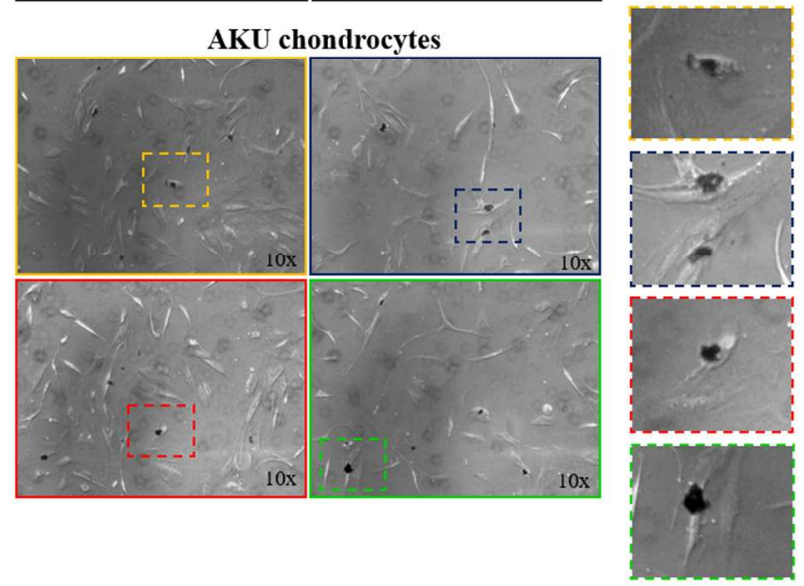

C
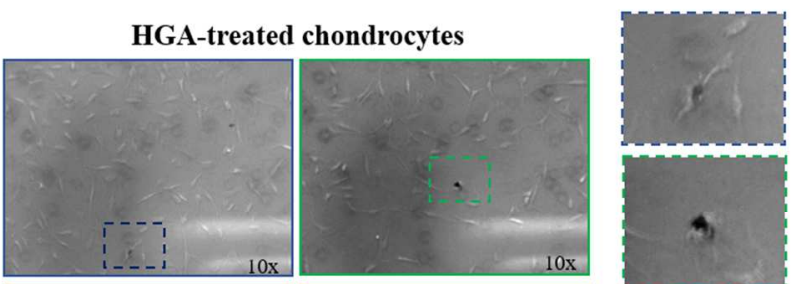

35

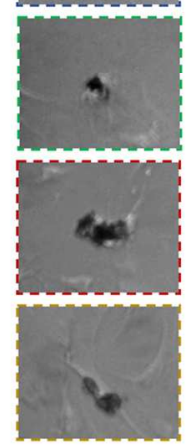

John Wiley \& Sons, Inc. 
A

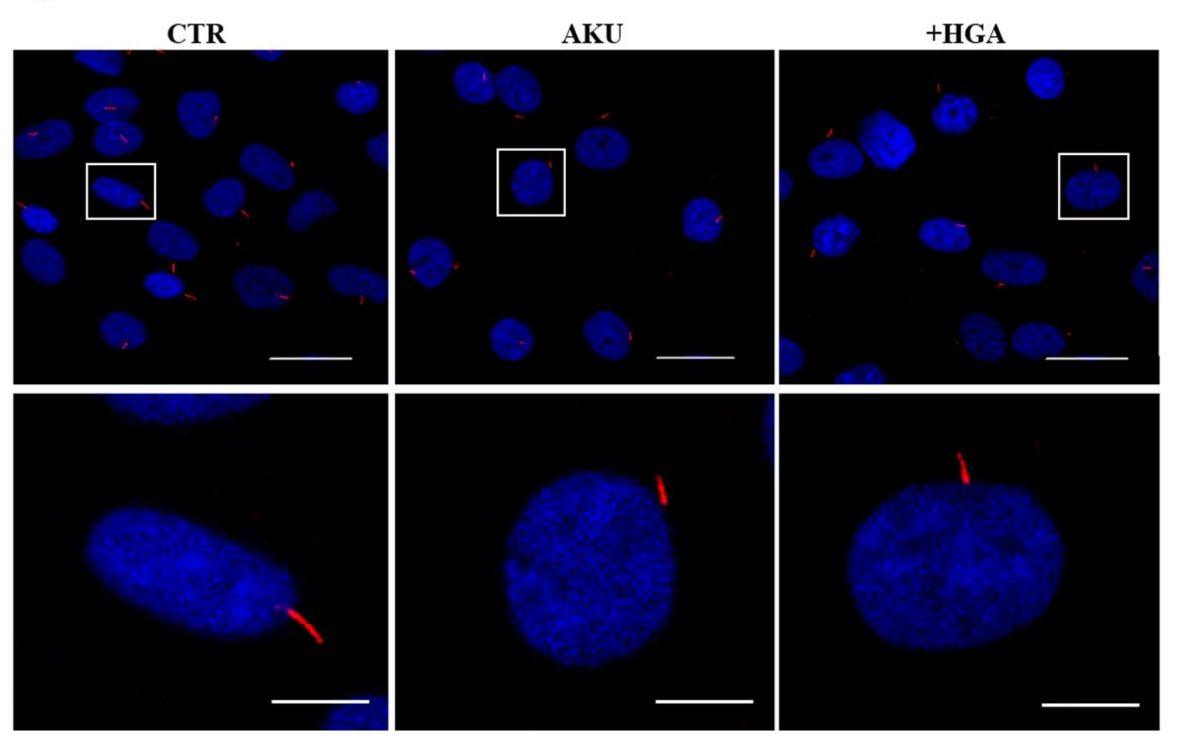

B

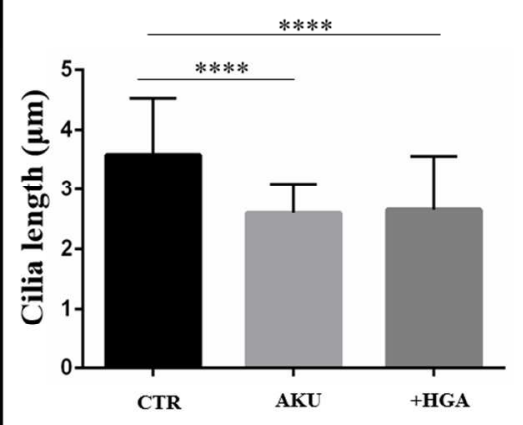

C

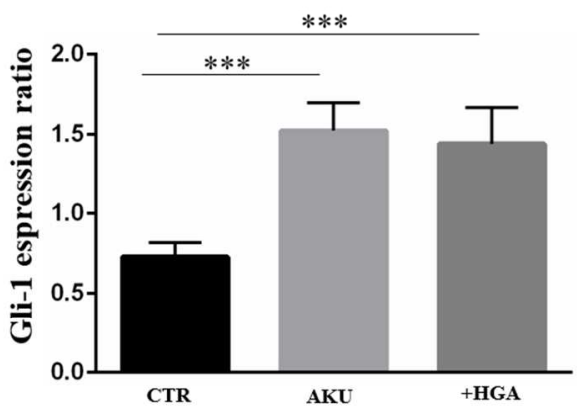

Gli-1 - 118 kDa

GAPDH- 37 kDa

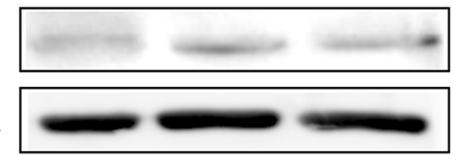

$197 \times 229 \mathrm{~mm}(150 \times 150$ DPI $)$

John Wiley \& Sons, Inc. 


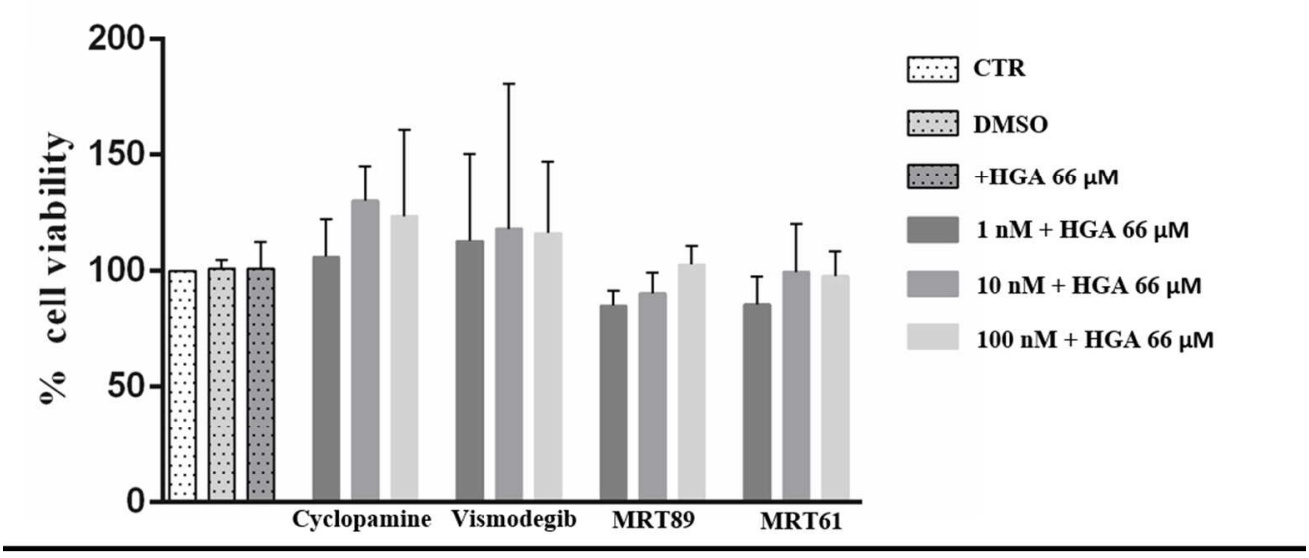

$184 \times 88 \mathrm{~mm}(150 \times 150 \mathrm{DPI})$

John Wiley \& Sons, Inc. 


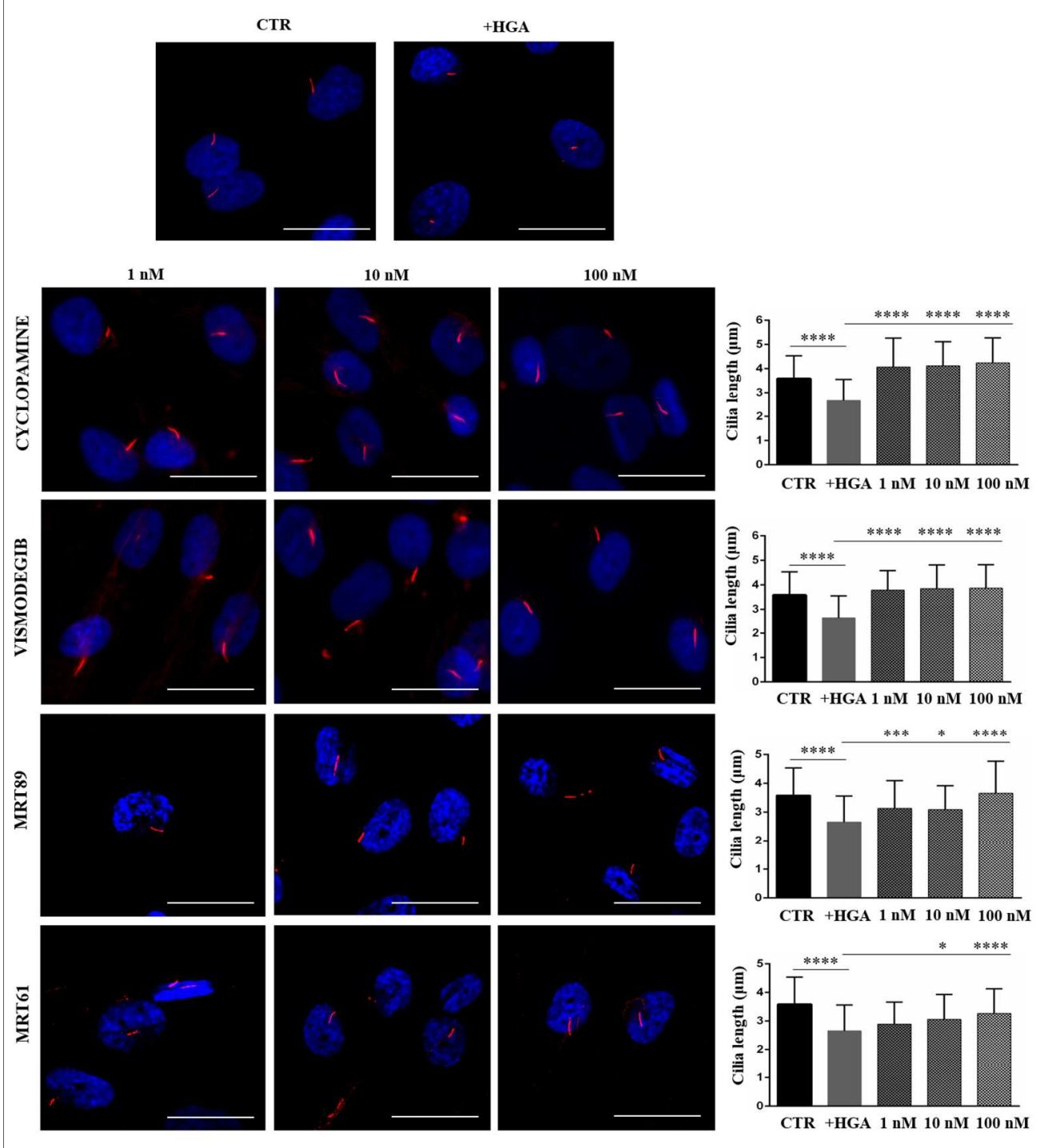

$245 \times 288 \mathrm{~mm}(150 \times 150 \mathrm{DPI})$

John Wiley \& Sons, Inc. 
Table 1 Primary cilia lengths and percentages of increase in respect to HGA-treated chondrocytes, after 24 hours of treatment with Smo antagonists.

\begin{tabular}{c|c|c|c}
\hline Drug name & $\begin{array}{c}\text { Treatment } \\
(\mathbf{n M})\end{array}$ & $\begin{array}{c}\text { Cilia length mean } \pm \\
\text { S.E.M. }(\boldsymbol{\mu m})\end{array}$ & $\begin{array}{c}\text { \% of cilia } \\
\text { length increase }\end{array}$ \\
\hline \multirow{3}{*}{ Cyclopamine } & 1 & $4 \pm 1.2$ & 53 \\
& 10 & $4.1 \pm 1$ & 55 \\
& 100 & $4.2 \pm 1$ & 60 \\
\hline \multirow{3}{*}{ Vismodegib } & 1 & $3.8 \pm 0.8$ & 43 \\
& 10 & $3.9 \pm 0.9$ & 45 \\
& 100 & $3.9 \pm 0.9$ & 46 \\
\hline & 1 & $3.1 \pm 0.9$ & 18 \\
MRT89 & 10 & $3.1 \pm 0.8$ & 17 \\
& 100 & $3.7 \pm 1.1$ & 38 \\
\hline & 1 & $2.9 \pm 0.8$ & 9 \\
MRT61 & 10 & $3 \pm 0.9$ & 15 \\
& 100 & $3.3 \pm 0.9$ & 24 \\
\hline
\end{tabular}

22 


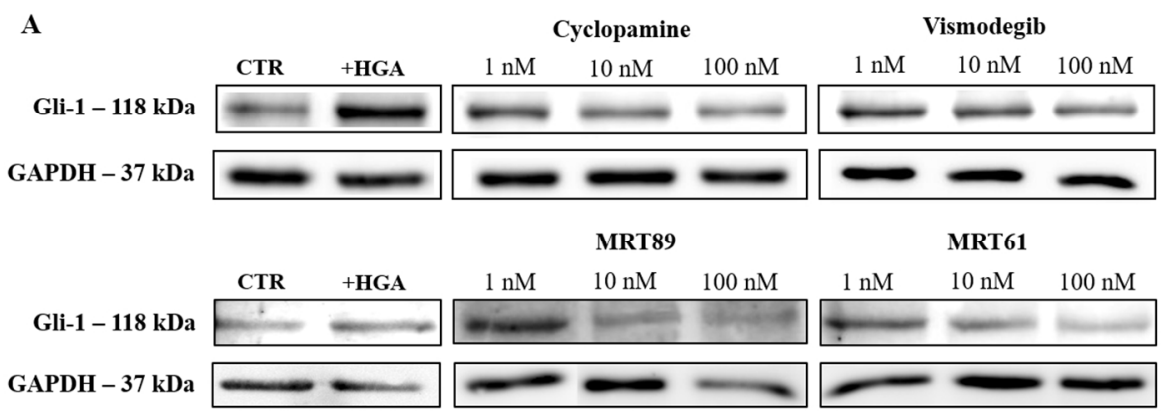

B
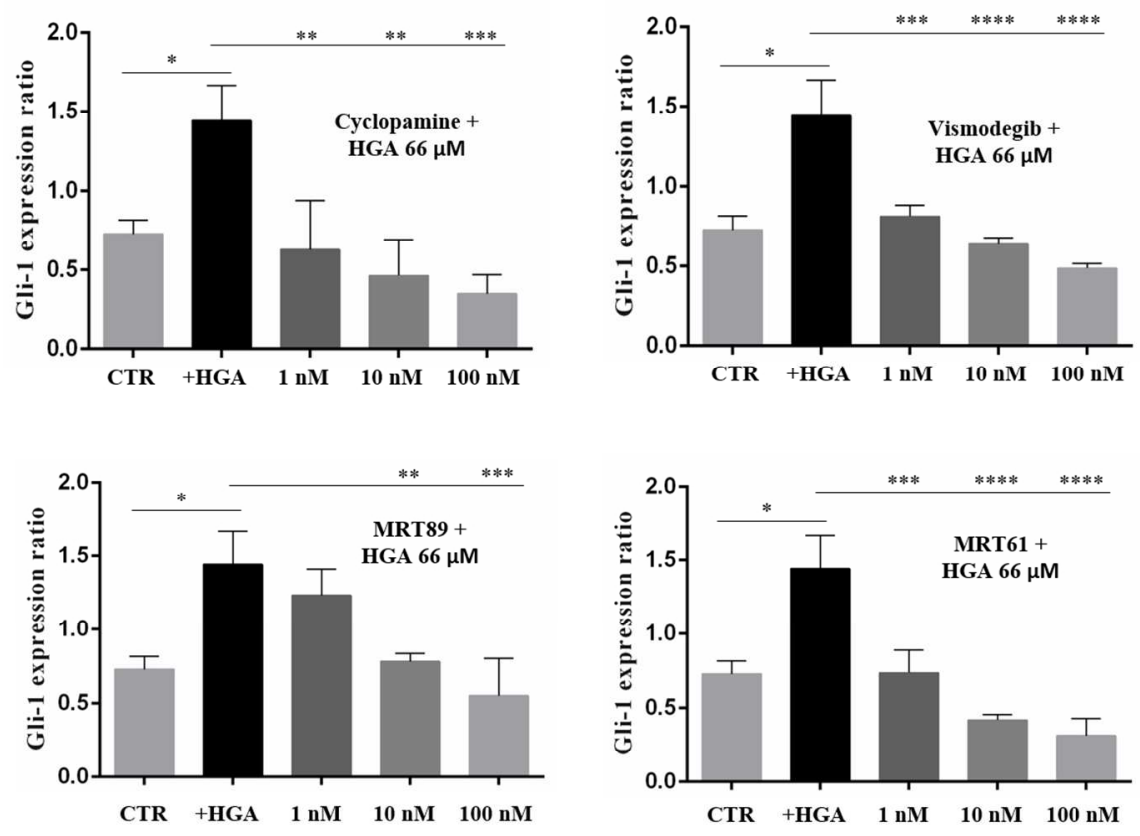

$224 \times 241 \mathrm{~mm}(150 \times 150 \mathrm{DPI})$

John Wiley \& Sons, Inc. 